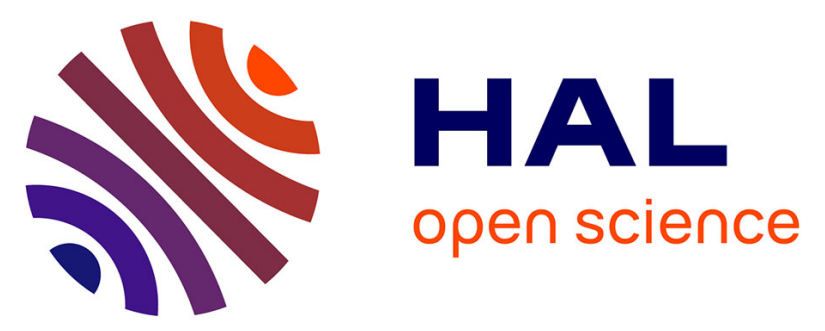

\title{
Discrete simulation of dense flows of polyhedral grains down a rough inclined plane
}

\author{
Emilien Azéma, Yannick Descantes, Nicolas Roquet, Jean-Noël Roux, \\ François Chevoir
}

\section{- To cite this version:}

Emilien Azéma, Yannick Descantes, Nicolas Roquet, Jean-Noël Roux, François Chevoir. Discrete simulation of dense flows of polyhedral grains down a rough inclined plane. Physical Review E: Statistical, Nonlinear, and Soft Matter Physics, 2012, 86 (3), pp.1-14. 10.1103/PhysRevE.86.031303 . hal-00737324

\section{HAL Id: hal-00737324 \\ https://hal.science/hal-00737324}

Submitted on 1 Oct 2012

HAL is a multi-disciplinary open access archive for the deposit and dissemination of scientific research documents, whether they are published or not. The documents may come from teaching and research institutions in France or abroad, or from public or private research centers.
L'archive ouverte pluridisciplinaire HAL, est destinée au dépôt et à la diffusion de documents scientifiques de niveau recherche, publiés ou non, émanant des établissements d'enseignement et de recherche français ou étrangers, des laboratoires publics ou privés. 


\title{
Discrete simulation of dense flows of polyhedral grains down a rough inclined plane
}

\author{
Emilien Azéma* ${ }^{1, \dagger}$ Yannick Descantes, ${ }^{1,}{ }^{\ddagger}$ Nicolas Roquet, ${ }^{1,} \S$ Jean-Noël Roux, ${ }^{2}$, ฯ and François Chevoir ${ }^{2,}{ }^{* *}$ \\ ${ }^{1}$ UNAM, IFSTTAR, Route de Bouaye, CS4, 44344 Bouguenais Cedex, France \\ ${ }^{2}$ Université Paris-Est, Laboratoire Navier (IFSTTAR, \\ Ecole des Ponts ParisTech, CNRS), Champs-sur-Marne, France
}

(Dated: September 4, 2012)

\begin{abstract}
The influence of grain angularity on the properties of dense flows down a rough inclined plane are investigated. Three-dimensional numerical simulations using the Non-Smooth Contact Dynamics method are carried out with both spherical (rounded) and polyhedral (angular) grain assemblies. Both sphere and polyhedra assemblies abide by the flow start and stop laws, although much higher tilt angle values are required to trigger polyhedral grain flow. In the dense permanent flow regime, both systems show similarities in the bulk of the material (away from the top free surface and the substrate), such as uniform values of the solid fraction, inertial number and coordination number, or linear dependency of the solid fraction and effective friction coefficient with the inertial number. However, discrepancies are also observed between spherical and polyhedral particle flows. A dead (or nearly arrested) zone appear in polyhedral grain flows close to the rough bottom surface, reflected by locally concave velocity profiles, locally larger coordination number and solid fraction values, smaller inertial number values. This dead zone disappears for smooth bottom surface. In addition, unlike sphere assemblies, polyhedral grain assemblies exhibit significant normal stress differences, which increase close to the substrate.
\end{abstract}

PACS numbers: 45.70.Mg, 81.05.Rm, 83.10-y, 83.80.Fg

\section{INTRODUCTION}

\section{A. State of the art: dense flow of spherical grains}

Research carried out over the past ten years have led to significant progress in the understanding of the rheology of dry granular materials in the dense regime [1-8]. Most studies, either experimental or by numerical simulation, have been carried out using circular two dimensional (2D) or spherical three dimensional (3D) grains. For such grains, the local rheological laws, i.e. the relations between normal stress $\sigma_{n}$, shear stress $\sigma_{t}$, shear rate $\dot{\gamma}$ and solid fraction $\nu$, have successfully been formulated, involving the dimensionless inertial number [3]:

$$
I=\dot{\gamma} d \sqrt{\frac{\rho}{\sigma_{n}}} .
$$

In (1), $d$ denotes the mean grain diameter and $\rho$ the mass density within the grains. Flow regimes can be classified according to $I$ values. Low $I$ values correspond to the quasi-static regime, the material behaving as an elastoplastic solid (critical state of soil mechanics) [9]. Conversely, high $I$ values correspond to the collisional regime, where grains interact through binary collisions that can

\footnotetext{
*now at LMGC, CNRS - Université Montpellier 2, cc 48, Place Eugène Bataillon, 34095 Montpellier cedex 05, France.

${ }^{\dagger}$ Electronic address: Emilien.Azema@univ-montp2.fr

$\ddagger$ Electronic address: Yannick.Descantes@ifsttar.fr

$\S$ Electronic address: Nicolas.Roquet@ifsttar.fr

"Electronic address: Jean-Noel.Roux@ifsttar.fr

**Electronic address: Francois.Chevoir@ifsttar.fr
}

be considered as instantaneous and decorrelated [10]. Between these two regimes, a dense flow regime exists for which the role of grain inertia cannot be neglected. Then, the material flows in a liquid rather than gaseous state. Grain displacements are strongly correlated. A contact network, which fluctuates both in space and time, percolates through the assembly. When $I$ increases, the mean contact duration decreases, the assembly dilates, and the force chains become smaller and scarcer.

The rheological laws may be described through the inertial number dependency of two dimensionless quantities, solid fraction $\nu$ and effective friction $\mu^{*}=\sigma_{t} / \sigma_{n}$. Simulations of 2D homogeneous shear flow [3] evidenced the following simple linear laws for solid fraction and effective friction coefficient, which provide good fit to the numerical data within interval $5 \cdot 10^{-3} \leq I \leq 0.15$ :

$$
\begin{aligned}
\nu(I) & =\nu_{\max }-a I \\
\mu^{*}(I) & =\mu_{\min }^{*}+b I .
\end{aligned}
$$

Maximum solid fraction $\nu_{\max }$ and minimum effective friction coefficient $\mu_{\min }^{*}$ can respectively be identified with the solid fraction $\nu_{c}$ and with the internal friction coefficient $\tan \phi$ in the critical state. The properties of the critical state generally depend only on grain geometry and contact friction coefficient [11]. Similar laws were identified in 3D simulations $[12,13]$, although power laws are reported with exponents below 1 for the dependence on $I$, as the quasistatic limit $I \rightarrow 0$ is approached. Coefficient $\mu^{*}(I)$ is also observed to cease increasing as $I$ reaches larger values, above a few times unity [5]. Eqs. (2)-(3) were successfully applied to 2D heterogeneous shear flows in annular cells [14]. They apply in homogeneous steady shear flows whatever the initial conditions. These laws have been generalized to cohesive granular materials [15] 
and to concentrated suspensions $[11,16]$, provided adequate dimensionless numbers are introduced to characterize viscous or cohesive effects. In general parameters $\nu_{\max }$ and $\mu_{\min }^{*}$, as well as coefficients $a$ and $b$, when a linear fit is used, depend on material characteristics $[3,17]$.

Dry granular material flows down a rough inclined plane have received wide attention in 2D [18] and 3D experiments $[5,19,20]$ and in 2D [20-23] and 3D [24] discrete numerical simulations. Truly $3 \mathrm{D}$ experiments have suggested and evidenced a tensorial form of the friction law $\mu^{*}(I)$ [5]. The plane bed must be sufficiently rough to affect the flow. Then, a dense steady and uniform flow is observed within a given range of tilt angle $\theta$ and flow thickness $H$ values. Starting from such a flow regime and slowly decreasing the tilt angle, the friction law (3) predicts gradual deceleration of the flow until a stop tilt angle $\theta_{\text {stop }}=\phi$ is reached, as well as gradual acceleration of the flow beyond a start tilt angle $\theta_{\text {start }}$ equal to the stop tilt angle. In fact, for various materials and bed roughnesses, a sudden flow stop is observed both experimentally and numerically for a tilt angle which is strongly influenced by the flow thickness, $H$ [19-22], and a sudden flow start is observed when the tilt angle is increased to reach a threshold value also highly dependent on the flow thickness $H$ the start tilt angle being higher than the stop tilt angle [25]. As a consequence, stop and start flow thicknesses $H_{\text {stop/start }}(\theta)$ may be defined, that can be well described by the following expression:

$$
H_{\text {stop } / \text { start }}(\theta)=B d \frac{\theta_{M}-\theta}{\theta-\theta_{m}},
$$

in which parameters $B$ (of order 1 ), $\theta_{m}$ and $\theta_{M}$ respectively express substrate surface roughness and flowing material dependent length $(B d)$ and limit tilt angles for thick and thin layers.

\section{B. Influence of grain shape}

The works mentioned above highlight that a minimalistic model of granular material reveals a complex generic rheology. However, this fact tends at the same time to hide the complexity arising from specific features such as the shape of particles.

Given a multitude of potential particle morphologies, a wide range of nontrivial effect were reported, both experimentally and numerically, mainly in static and quasistatic conditions [26-42]. Only very recently did systematic studies of elongated [43-50] or nonconvex [51, 52] particles report unusually high or low packing fractions, while the shear strength is observed to grow as the shape increasingly deviates from the disk or the sphere. The shear strength in assemblies of regular n-sided polygons increases with angularity $2 \pi / n$ and saturates at a maximum, whereas the packing fraction declines towards a plateau [53]. In all cases, the increase of shear strength with a suitably defined non-sphericity parameter may be attributed to the increasing frustration of particle rotations [54].

The flows of smooth-shaped non-spherical particles (e.g., spheroid and cuboids) were also studied in various geometries $[28,29,33,40,55-57,57-66]$. Those works reported strong shear strength increases away from the spherical shape. At the same time, the increase of the aspect ratio of the particles leads to an increase in solid fraction near the wall and to a lower central solid fraction [67]. Remarkably, faster silo discharges are observed for elongated particles than for circular or spherical ones [59].

The flows of particles of more complex shape were studied experimentally by Borzsonyi et al. [62, 63], who, interestingly, reported that the effective friction increases with bed roughness, and that avalanches of non-spherical particles have a higher velocity than for spherical particles. Numerically, it is only recently that more complex shapes, such as polyhedra, have been used to simulate silo discharge flows [68-71]. It has been shown that the increase of the angularity of the particles reduces the mass flow rate from the hopper.

Nevertheless, no results have been reported yet on the effect of particle shape on macroscopic laws. Such a study involves technical difficulties, related to contact detection and force calculation between particles of arbitrary shape [72-76], and also requires extensive simulation campaigns to systematically explore sufficiently wide ranges of material states. It is nevertheless felt necessary to understand to what extent local laws evidenced for spherical grains (flow threshold, rheological laws) still apply to angular shapes.

\section{Engineering concerns about grain angularity}

The angularity of aggregates -i.e., granular materials used in construction and civil engineering - has long been recognized as an issue of great practical importance. More than $85 \%$ of the volume of road pavement materials is occupied by aggregate grains, either unbound or mixed with a bituminous binder. Of the 3 billion tons of aggregate produced each year in Europe (31 countries, including Russia), about 40\% come from alluvial deposits [77], and are made of rounded shaped grains or gravels. This lack of angularity induces poor performance in terms of pavement surface skid resistance [78] and rutting of asphalt mixes $[79,80]$ or unbound [81] structural layers.

In order to avoid excessive amounts of rounded gravels in road building materials, as direct visual inspection of grain surfaces is slow and inaccurate, a test based on flow rate measurement at the outlet of a vibrated vertical chute is customarily employed [82]. Even though the flow rate is also influenced by rock texture, aggregate grading and flakiness index $[83,84]$, it is regarded as characterizing the angularity of the grains, as it notably increases for rounded particles as compared to gravels with a high percentage of crushed and broken surfaces. Motivated 
by the search for simpler, more reproducible and better characterized rheological tests than vibrated chute flows, rough inclined plane experiments recorded the effects of gravel angularity on steady-state flow rates [85], in good correlation with vibrated vertical chute results [86]. The dense permanent flow regime is shifted to larger slope angles for angular gravels as compared to rounded ones, but results are qualitatively similar for slope angle and layer thickness dependencies, and flow thresholds are still fitted by relation (4), in which parameters $\theta_{m}, \theta_{M}$ and $B$ depend on grain angularity.

\section{Objectives. Outline of the paper}

This study exploits the unique ability of discrete simulations of granular assemblies to carry out "numerical experiments" in ideally controlled conditions, and to provide detailed information on the internal state of the tested material. In order to investigate the influence of grain angularity on the properties of dense flows down a rough inclined plane, formulated in intrinsic rheological terms, simulations using either angular or spherical grains are performed in parallel and their results are systematically compared. After the simulated system are described in Sec. II, Sec. III reports on the flow diagrams and on the profiles of various quantities in dense steady flows. Then Sec. IV identifies constitutive laws. Sec. V summarizes our results and draws some perspectives.

\section{SIMULATED SYSTEM}

\section{A. Shape of grains}

The simulated system is a three dimensional dense assembly of $n$ grains of mass density $\rho$, interacting by contact forces involving two dissipative mechanisms, friction (microscopic friction coefficient $\mu$ ) and totally inelastic collisions. Two grain shapes are studied, spherical grains of average diameter $d$ and polyhedral grains of average characteristic dimension $d$. The polyhedral geometry (Fig. 1) is that of a pinacoid, with eight vertices, fourteen edges and eight faces. This pohyhedron

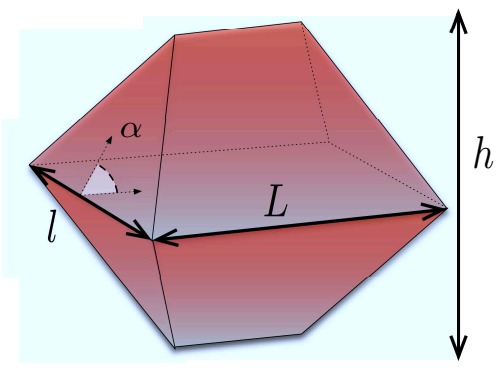

FIG. 1: (Color online) Model polyhedral grain: pinacoid.

has three planes of symmetry and is determined by four parameters: length $L$, width $l$, height $h$ and angle $\alpha$. According to an extensive experimental study with various rock types [87], the pinacoid gives the best fit among simple geometries for an aggregate grain. In order to have the same aspect ratio for both grain geometries, the pinacoid dimensional parameters are taken identical $(L=l=h)$, with the characteristic dimension $d$ expressed as $d=\sqrt{L^{2}+l^{2}}$. In addition, angle $\alpha$ is set to $60^{\circ}$ (so that the grain volume is approximately equal to $0.143 d^{3}$, around $1 / 3$ of the volume of a sphere). Besides, grain diameters (or characteristic dimensions) are uniformly distributed between $d .(1-\varepsilon)$ and $d .(1+\varepsilon)$, with $\varepsilon=0.1$ to prevent both crystallization and segregation phenomena.

Previous studies showed that, in the rigid contact limit - say, when typical contact deflections do not exceed $10^{-4} d$ - the rheology of granular flows does not depend on contact elasticity $[3,4,13,17,88-90]$. Given usual values of elastic modulus and stresses, this applies to most natural and experimental granular flows, and we therefore deal with perfectly rigid grains.

\section{B. Flow configuration. Sample preparation.}

The sample geometry is that of a parallelepiped (Fig. 2) limited in the $z$ direction by a fixed rough bottom wall and a free surface at the top, with periodic boundary conditions applied in the flow direction $y$ and in the transverse direction $x$. Thanks to these periodic conditions, the flow structure is not affected by lateral walls, contrary to experiments [91] and infinitely long chutes can be simulated using a finite number of grains. The lengths of the parallelepiped $\left(L_{y}=30 d\right.$ and $\left.L_{x}=26 d\right)$ are large enough to avoid size effects [18, 22, 24]. The initial configuration is prepared according to the following geometrical deposition protocol [92]: spherical grains are sequentially dropped along $z$ in the simulation box, and each grain stops on the free surface made of the preceding layer of grains (or on the rough bottom wall for the first layer of grains), so that each new grain relies on three grains chosen in order to minimize its altitude $z$. In the case of polyhedral grains, a pinacoid with the largest possible characteristic dimension $d$ is subsequently introduced in each sphere and randomly oriented.

For both studied grain shapes, a specific rough substrate is prepared, consisting of a plane bottom wall on which grains (identical to the flowing ones) are glued. The rough substrate is constructed by geometrical deposition of three layers of grains according to the preceding procedure. The lowest one is in contact with the bottom plane, while the highest grains are removed in order to keep a thickness of the rough bottom of approximately $1.5 d$. In the case of polyhedral grains, an intermediate phase consisting of grain deposition under gravity is needed prior to the elimination of the highest grains, as the geometrical deposition protocol does not lead to mechanical equilibrium. In the following, $z=0$ 
$\longrightarrow \quad$ applied in order to initiate the flow, the bottom rough

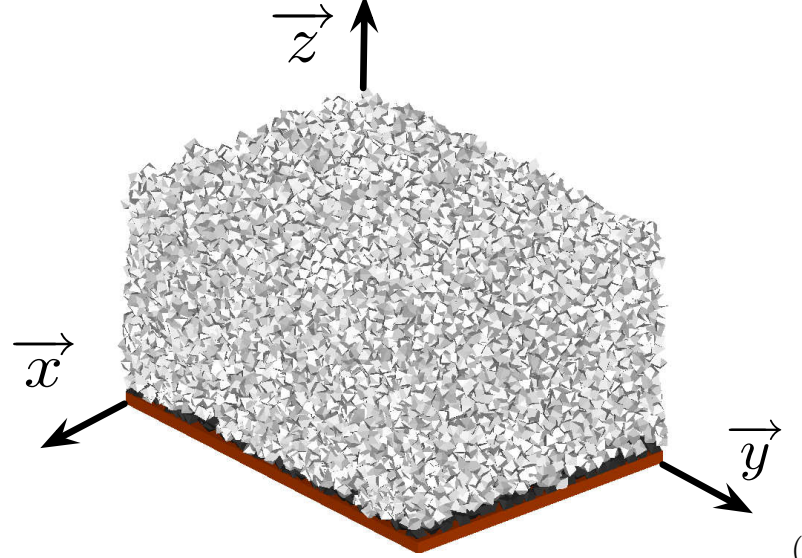

surface being now inclined at angle $\theta$ with respect to the horizontal plane. According to the geometrical deposition protocol, spheres are in contact when the gravity is applied, while pinacoids are still free of contact inside their virtual spherical envelope. However, all features of initial configurations are forgotten in the steady state flows investigated in the present study.

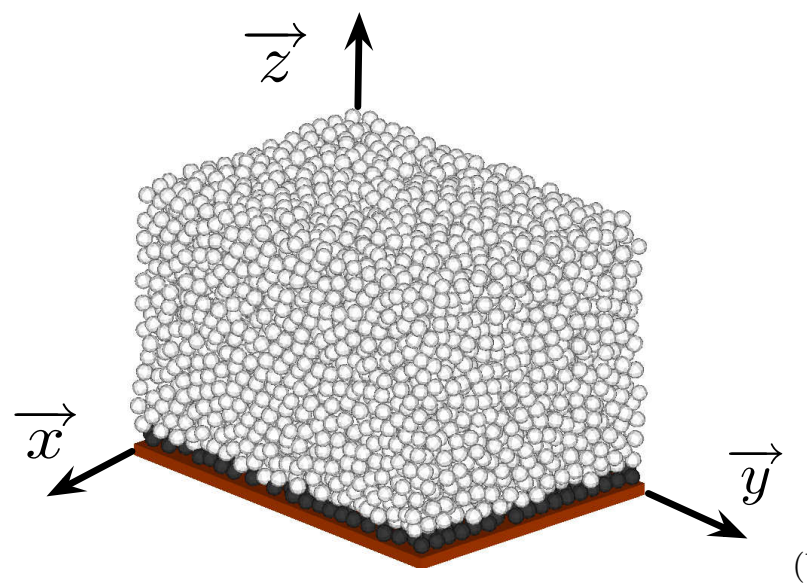

(a)

FIG. 2: (Color online) Samples of 19000 polyhedra (a) and 6900 spheres (b).

corresponds to a distance $d$ from the plane bottom wall. Fig. 3 shows an image of the substrate used for polyhedral particles. In the next preparation step, $n$ free grains

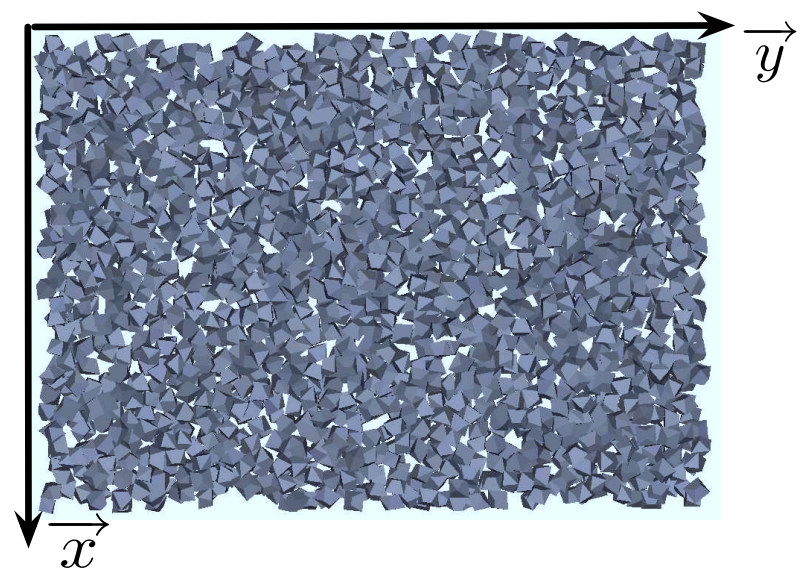

FIG. 3: (Color online) Rough substrate obtained on gluing pinacoids to a plane, seen from above.

are geometrically deposited onto this substrate, by the same procedure. Finally, gravity $\vec{g}(0, g \sin \theta,-g \cos \theta)$ is

\section{Contact dynamics method}

The numerical simulations are carried out using the contact dynamics (CD) method [76, 93-95]. The method integrates the equations of motion of a collection of rigid objects with contact interactions, involving Coulomb friction and dissipative collisions. It relies on an implicit time-stepping scheme in which the contact laws are prescribed as complementarity relations between momentum transfers at contacts within one time step and relative velocities, in such a way that enduring contacts and instantaneous collisions are dealt with on an equal footing. These laws introduce restitution coefficients (a normal one, $e_{N}$ and a tangential one, $e_{T}$ ) and a coefficient of sliding friction $\mu$ such that the Coulomb inequality $f_{t} \leq \mu f_{n}$ is enforced on tangential $\left(f_{t}\right)$ and normal $\left(f_{n}\right)$ force components at a contact point, with sliding relative motion only allowed when the equality is fulfilled. The contact laws are nonsmooth, as they express no functional dependence between contact forces and relative velocities. Iterative methods are used to determine both forces and velocities at the end of each time step, simultaneously. The CD method proved apt to deal with dense flows $[4,21,96]$ as well as with quasi-static plastic deformation [48, 75, 97-101]. One advantage of the CD method over more conventional molecular dynamics (MD) is that time needs not be finely discretized, as the very small space and time scales associated with contact deflection and collisions are overlooked. Some comparisons of CD and MD methods can be seen in [102, 103]. In our simulations the time step is fixed to $5.10^{-3} t_{0}$, with $t_{0}=\sqrt{d / g}$, the characteristic free fall time of a grain over height $d$ under gravity $g$.

To simulate polyhedral particles we use the LMGC90 software implementation $[76,104,105]$ of the CD method, which exploits a 3D contact detection algorithm described in detail in $[72,76,105]$, and deals with the various types of contacts illustrated in Fig. 4 as sets of one to three independent point contacts. Vertex to face contacts, and edge to edge ones, are simple point contacts. Edge to face contacts are dealt with as double contacts, or pairs of contacts points, each one being applied the contact law. Face-to-face contacts are decomposed into triangular regions, each treated as a triple contact (a triplet of contact points). Two forces are calculated for edge to face contacts and three forces for face to face contacts, but only the net resulting force and its point of application are physically meaningful. 


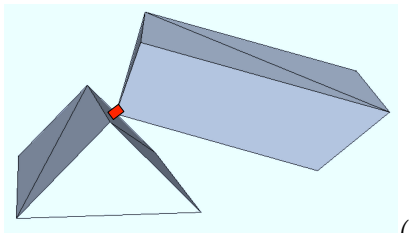

(a)

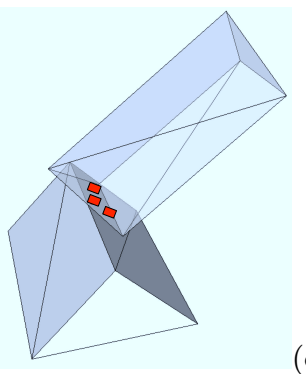

(c)

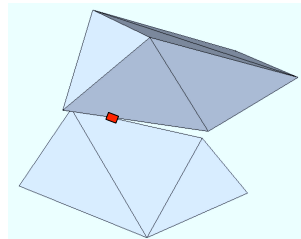

(d)

FIG. 4: (Color online) Different types of contacts between two polyhedra: (a) vertex to face; (b) edge to face; (c) face to face; (d) edge to edge.

\section{Material and system parameters}

For spheres, the number of grains in the simulated cell, $n$, varies between 1100 and 6900 , so that $H / d$ varies between 5 and 18. For pinacoids, $n$ varies between 7100 and 19000 , so that $H / d$ varies between 6 and 14 . Because of the dilation of the material, height $H / d$ will slightly increase when $\theta$ increases.

TABLE I: Simulated systems. Number of grains $(n)$, horizontal dimensions of simulation cell $\left(L_{y} / d\right.$ and $\left.L_{x} / d\right)$, friction $(\mu)$ and restitution $\left(e_{N, T}\right)$ coefficients.

\begin{tabular}{lccccc}
\hline \hline Grain & $n$ & $L_{y} / d$ & $L_{x} / d$ & $\mu$ & $e_{N, T}$ \\
\hline Spheres & $1100-6900$ & 30 & 26 & 0.4 & 0 \\
Pinacoid & $7100-19000$ & 30 & 26 & 0.4 & 0 \\
\hline \hline
\end{tabular}

The simulated system parameters are listed in Tab. I. Unless specified differently, the friction coefficient, $\mu_{W}$, in contacts between the flowing particles and the rough substrate is equal to $\mu$. In the sequel, results are given in dimensionless form on expressing lengths and times in respective units of $d$ and $t_{0}$ (defined in Sec. IIC), while the stress scale is given by $\rho g d$.

\section{FLOW CHARACTERISTICS}

Dense permanent flows down a rough inclined plane being expected only for a finite range of parameters $H$ and $\theta$, the phase diagram $(\theta, H)$ with the flow thresholds is first determined (Sec. III A). Then, dense permanent flow regimes are analyzed in detail through the profiles of various quantities (solid fraction, coordination number, velocity, stress components and inertial number) in Sec. III B. We also investigate some specific properties of the polyhedral particle layers flowing close to the substrate in (Sec. III C).

\section{A. Phase diagram}

We typically observe two kinds of states: in the flowing states, the ratio of the total kinetic energy of the flowing grains to their potential energy in the gravity field is of order 1 with relative fluctuations of order 0.1 (increasing close to stop). This ratio drops below $10^{-4}$ in the no-flow state.

Fig. 5a shows two pairs of curves characterizing the flow initiation $\left(H_{\text {start }}\right)$ and cessation $\left(H_{\text {stop }}\right)$ calculated for spheres and pinacoids, as functions of tilt angle $\theta$. To determine these curves a number $n$ of grains is chosen, corresponding to a typical flow height $H$, and a tilt angle $\theta$ is imposed, such that the material flows. Then, the tilt angle is gradually reduced by steps of $0.5^{\circ}$ down to a critical value $\theta_{\text {stop }}$ corresponding to flow stop. Thickness $H$ is then measured in the no-flow state. Next, the tilt angle is slowly increased by steps of $0.5^{\circ}$ until a critical value $\theta_{\text {start }}$ corresponding to flow start is reached, whence a measurement of the corresponding layer thickness $H$. This procedure is repeated for different numbers $n$ of grains and leads to various $\theta_{\text {stop }}(H)$ and $\theta_{\text {start }}(H)$ thresholds, from which $H_{\text {stop }}(\theta)$ and $H_{\text {start }}(\theta)$ curves are built.

In agreement with [19], Fig. 5a shows that the flow stop and initiation curves calculated for spheres are fitted by Eq. 4, for pinacoids as well as for spheres, with a shift towards larger values of $\theta$ for the angular grains. The values of constant $B$, and angles $\theta_{m}$ and $\theta_{M}$ are given in Tab. II. On switching from spheres to pinacoids, the three parameters significantly increase, as $B$ nearly doubles while threshold angles $\theta_{m}$ and $\theta_{M}$ are respectively shifted by approximately $13^{\circ}$ and $16^{\circ}$.

Fig. 5b displays the flow threshold curves measured by [19] for glass beads and by [85] for angular aggregates (roughness made of grains similar to the flowing ones). We observe a good qualitative agreement between these experimental values and the present simulation results. Furthermore, in the case of spheres, the calculated values are also consistent with the 3D simulations results of [24].

TABLE II: Values of the parameters $B, \theta_{m}$ and $\theta_{M}$ for $H_{\text {stop }}(\theta)$ and $H_{\text {start }}(\theta)$ for polyhedra and spheres.

\begin{tabular}{lccc}
\hline \hline & $B / d$ & $\theta_{m}$ & $\theta_{M}$ \\
\hline$H_{\text {stop }}(\theta)$ (Spheres) & 2 & $19^{\circ}$ & $31^{\circ}$ \\
$H_{\text {stop }}(\theta)$ (Polyhedra) & 3.5 & $32^{\circ}$ & $47^{\circ}$ \\
$H_{\text {start }}(\theta)$ (Spheres) & 2.5 & $20^{\circ}$ & $31^{\circ}$ \\
$H_{\text {start }}(\theta)$ (Polyhedra) & 4.2 & $32^{\circ}$ & $49^{\circ}$ \\
\hline \hline
\end{tabular}


In Fig. 7 coordination number profiles $Z_{c}(z)$ for polyhedra (a) and for spheres (b) are plotted for different values of $\theta . Z_{c}$ is the mean number of contacts per grain. For polyhedra, a contact is counted just once whatever its type (simple, double or triple). $Z_{c}$ appears to be nearly uniform in the bulk of the assembly and it decreases when the tilt angle increases. For spheres, $Z_{c}$ decreases from about 3.6 for $\theta=21^{\circ}$ to about 2 for $\theta=28^{\circ}$, which falls within the range of values observed by [24]. The maximum value, 3.6 , is still somewhat below the minimum coordination number (about 4 , see [107]) in equilibrated bead packs, just like coordination numbers below 3 were reported in [3]. The quasistatic limit of $Z_{c}$ requires extremely small $I$ values to be accurately approached. Moreover, values of $Z_{c}$ in dense flows remain somewhat sensitive to restitution coefficients and elastic contact stiffness $[3,24]$, even though constitutive relations are not. For polyhedra, $Z_{c}$ decreases from roughly 3.3 for $\theta=35.7^{\circ}$ to about 2.7 for $\theta=38^{\circ}$, but it always increases significantly close to the substrate, where the solid fraction increases.
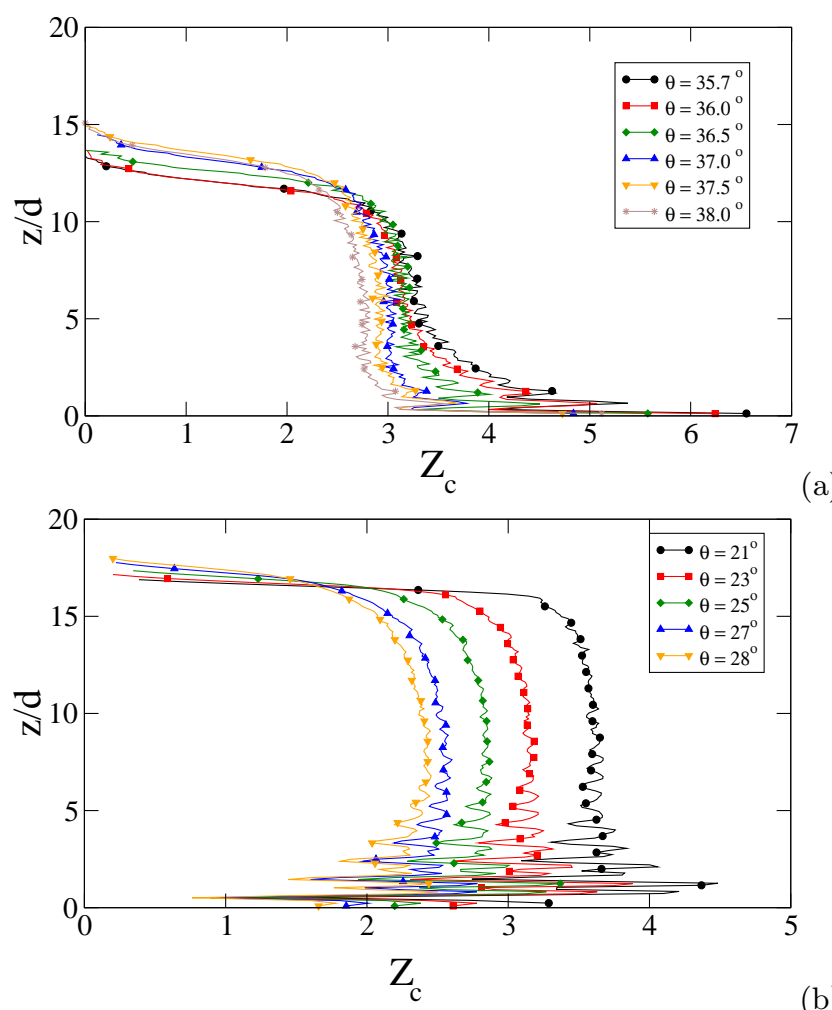

(a)

FIG. 7: (Color online) Coordination number profiles for polyhedra (a) and spheres (b).
Fig. 8 shows velocity profiles $v_{y}(z)$ for polyhedra (a) and for spheres (b) as a function of tilt angle $\theta$. In both cases, no sliding is observed at the rough bed interface, and the velocity profiles are convex a few layers away from the rough bed. In addition, shear rate $\dot{\gamma}$ decreases to zero at the rough bottom in the case of polyhedra, which is not the case for spheres. Thus, velocity profiles are totally convex for spheres, whereas they are concave close to the rough bed for polyhedra. Besides, in the case of polyhedra, scaled velocities are significantly higher than for spheres (by a factor of 5) just above the flow start threshold (tilt angle $35,7^{\circ}$ and $36^{\circ}$ ).

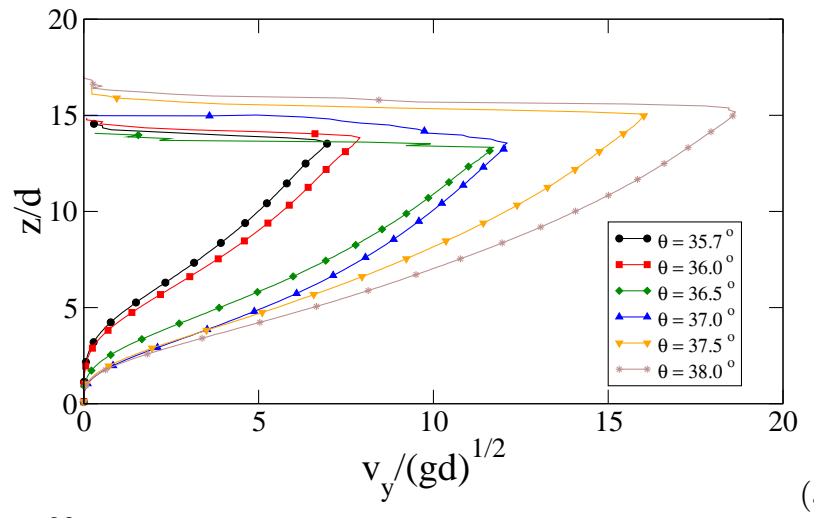

(a)

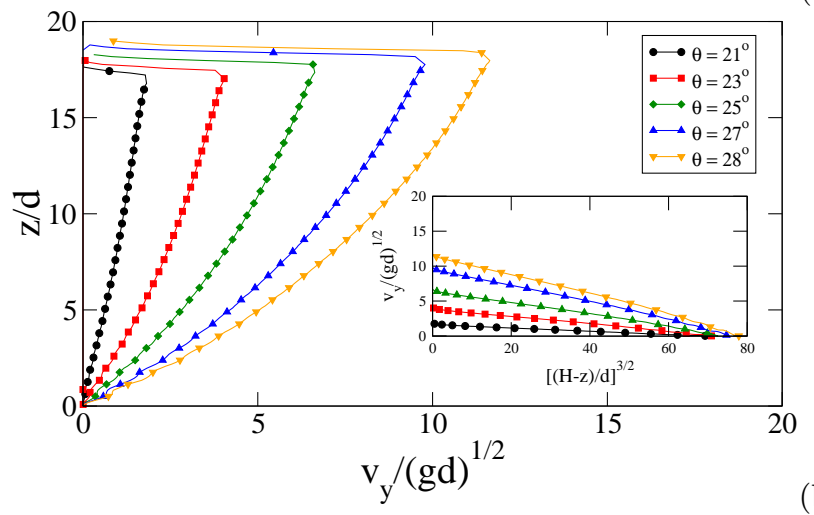

(b)

FIG. 8: (Color online) Velocity profiles for polyhedra (a) and spheres (b) - insert: test of Bagnold's rheology.

\section{Angular velocity}

Fig. 9 shows mean rotation velocity profiles $\omega_{x}(z)$ for polyhedra (a) and for spheres (b) as a function of tilt angle $\theta$. Like for the previous three profiles, a difference can be observed close to the rough bed between spheres and polyhedra. In both cases rotation velocities tend to decrease away from the rough bed, but angular velocities first increase with $z$ (for $z / d \leq 5$ ) for polyhedral grains. It was previously shown that the relation $\omega_{x}=-\dot{\gamma} / 2$ is very well satisfied in various shear configurations [14, $15,18,20,22]$. In Fig. 9, the shear rate is evaluated 
using a polynomial fit of the velocity profile (represented as symbols). This relation remains valid for spheres and polyhedra across the full assembly thickness.

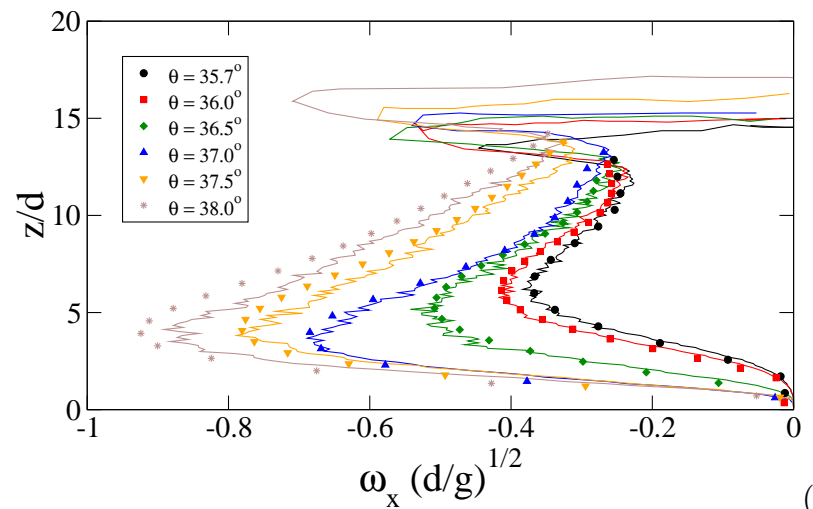

(a)

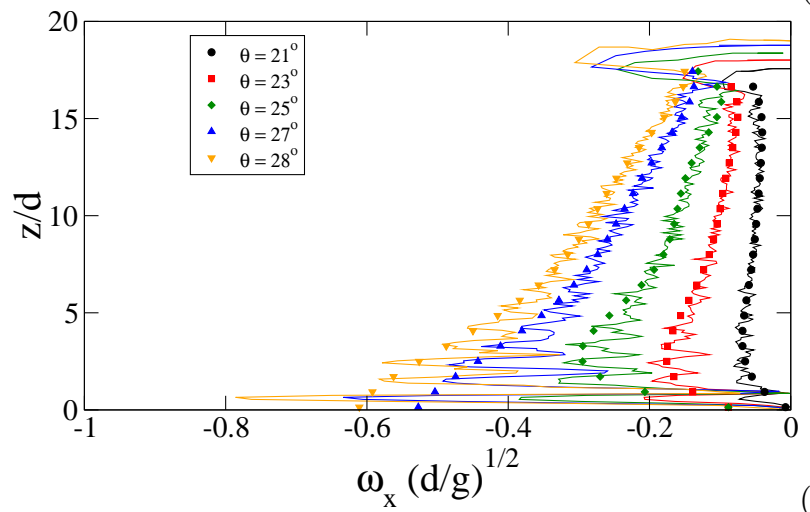

FIG. 9: (Color online) Angular velocity profiles for polyhedra (a) and spheres (b). Comparison with $-\dot{\gamma} / 2$ (symbols).

\section{5. $\quad$ Stress components}

Fig. 10 shows mean stress profiles $\sigma_{z z}(z)$ and $\sigma_{y z}(z)$ for polyhedra (a) and for spheres (b) for various tilt angle $\theta$. In dense flows the stress tensor $\sigma$ is dominated by the contribution of contact forces. $\overline{\overline{T h}}$ contribution of grain velocity fluctuations remains very small (about 20 times smaller for polyhedra) compared to that of contact forces in all the simulations considered here [3, 22], so that we shall not discuss it in the following. We adopt the symmetrized expression suggested by Moreau [108]. Otherwise, asymmetries of the order of $50 \%$ are observed for polyhedra. Local expressions are calculated within each slice $z$ to $z+\delta z$ from the contact forces at any contact point $c$ inside the slice, whence the formula:

$$
\underline{\underline{\sigma}}(z)=\frac{1}{L_{x} L_{y} \delta z} \operatorname{Sym}\left(\sum_{z \leq c \leq z+\delta z} \vec{f}^{c} \otimes \vec{r}^{c}\right),
$$

in which $\vec{f}^{c}$ is the contact force (or the sum of contact forces in case of double or triple contact) exerted on grain $j$ by grain $i$ and $\vec{r}$ is the vector pointing from the center of mass of grain $i$ to the center of mass of grain $j$. Also observe that, since the CD method determines contact impulses $\vec{P}^{c}$ at each time step, the contact forces are given by $\vec{f}^{c}=\vec{P}^{c} \times \Delta t$.

As a check, we compare our calculations with the predictions of the momentum balance equations in steady uniform flow:

$$
\left\{\begin{array}{l}
\sigma_{z z}(z)=\rho g \cos \theta \int_{z}^{\infty} \nu(t) d t \\
\sigma_{y z}(z)=-\rho g \sin \theta \int_{z}^{\infty} \nu(t) d t
\end{array}\right.
$$

The insert in Fig. 10 shows excellent agreement. In such
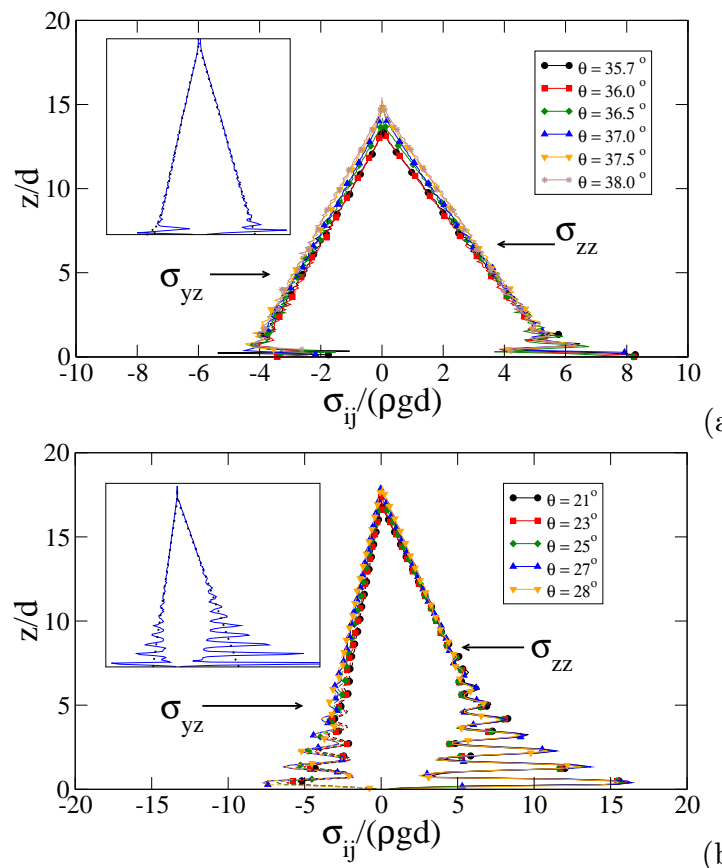

(a)

FIG. 10: (Color online) Stress component profiles for polyhedra (a) and spheres (b). In both cases, the insert compares calculated stresses (plain curves) and those predicted by (6) (dashed lines) for $\theta=36^{\circ}$.

a steady uniform flow the effective friction coefficient is defined as $\mu^{*}=-\sigma_{y z} / \sigma_{z z}$ and is equal to $\tan \theta$. Fig. 11 focuses on mean normal stress profiles $\sigma_{x x}(z), \sigma_{y y}(z)$ and $\sigma_{z z}(z)$ for polyhedra with $\theta=36^{\circ}$ (a) and for spheres with $\theta=21^{\circ}$ (b).

For spheres, one observes $\sigma_{y y} \simeq \sigma_{z z}$ while $\sigma_{x x}$ is slightly (typically by 15\%) smaller in the bulk of the material, in full agreement with [24]. The layer structuring perpendicular to the z-axis, close to the rough bottom, induces oscillations in the stress profiles, as most contacts are located at regular intervals along the $\mathrm{z}$ axis, and their favored orientation is correlated to $z$. If $l$ denotes the order number of a layer (ranging from 1 close to the rough wall to $H / d$ at the top free surface), contacts between particles of the same layer are more frequent in planes $z=(l-1 / 2) d$, while interlayer ones tend to occur at $z=(l-1) d$. As a consequence, $\sigma_{z z}(z)$ reaches maxima 
as $z$ is an integer multiple of $d$, whereas $\sigma_{x x}(z)$ and $\sigma_{y y}(z)$ reach their maxima as $z$ is an odd multiple of $d / 2$.

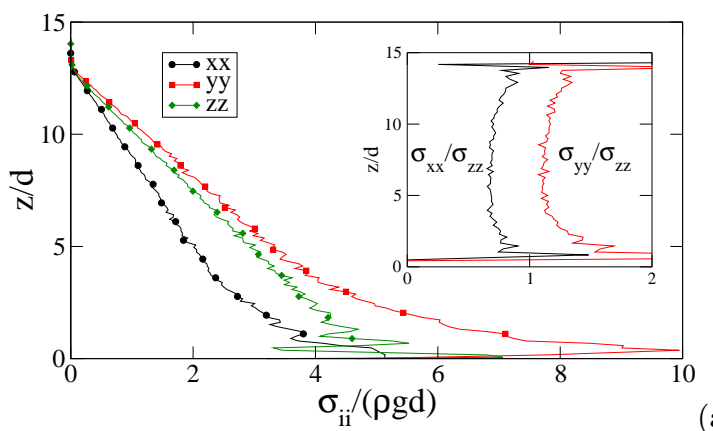

(a)

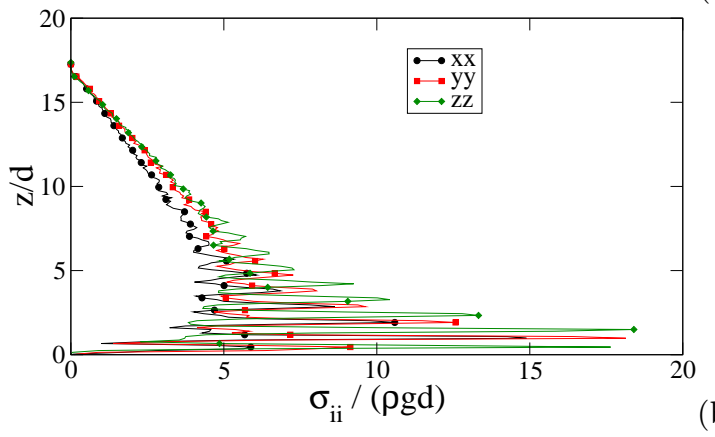

(b)

FIG. 11: (Color online) Normal stress profiles in the $\mathrm{x}, \mathrm{y}$ and $\mathrm{z}$ directions for polyhedra with $\theta=36^{\circ}$ (a) and spheres with $\theta=21^{\circ}(\mathrm{b})$.

For polyhedra, we observe differences between normal stress components $\sigma_{x x}, \sigma_{y y}$ and $\sigma_{z z}$. In the bulk of the material, $\sigma_{y y}$ is larger than $\sigma_{z z}$ by about $15 \%$ and $\sigma_{x x}$ is smaller than $\sigma_{z z}$ by roughly $20 \%$ (see insert in Fig. 11a).

\section{Inertial number}

Fig. 12 plots inertial number profiles $I(z)$ (with $\sigma_{n}=$ $\sigma_{z z}$ in Eq.1) for polyhedra (a) and for spheres (b) for different values of tilt angle $\theta$. We observe that $I$ is uniform for spheres, except close to boundaries, as larger $I$ values are recorded in an agitated thin layer near the free surface and as local layerwise structuring affects the material close to the substrate. For polyhedra, however, discarding the immediate vicinity of the free surface, two regions should be distinguished. While $I$ is nearly uniform above $z=5 d$, it is a growing function of $z$ below.

For spheres, since the solid fraction is nearly uniform along $z$, one may estimate:

$$
\sigma_{z z}(z)=\rho g \nu(H-z) \cos \theta
$$

where $\nu$ slightly depends on $\theta$ (dilatancy effect). Then, since the inertial number is also nearly uniform along $z$, we conclude that the stress components satisfy the Bagnoldian scaling $\sigma_{i j} \propto \dot{\gamma}^{2}$ [109]. We may even deduce that the velocity profile verifies:

$$
v_{y}(z)=\frac{2 I(\theta) \sqrt{\nu(\theta) g \cos \theta}}{3 d}\left(H^{3 / 2}-(H-z)^{3 / 2}\right) .
$$
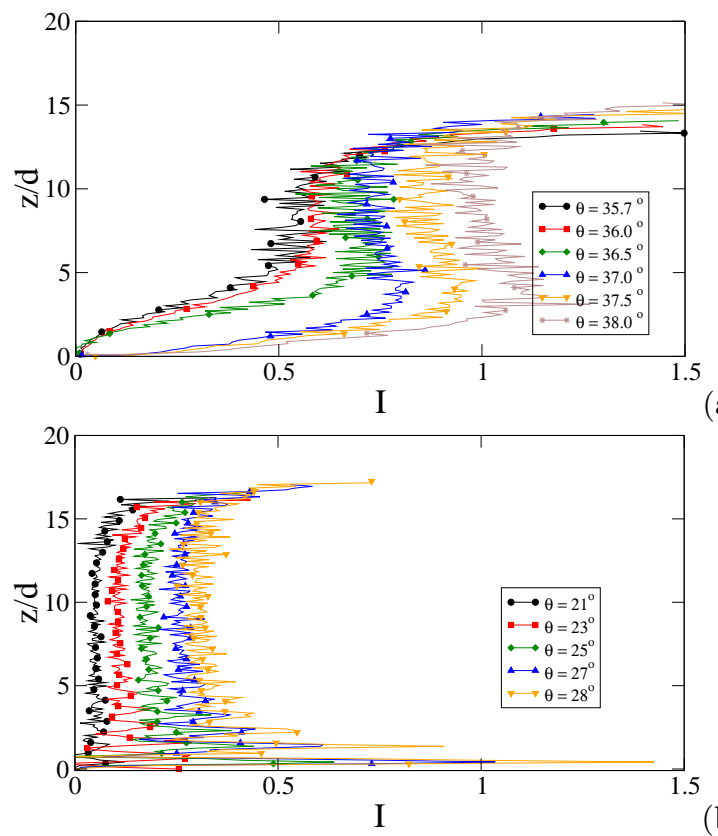

FIG. 12: (Color online) Inertial number profiles for polyhedra (a) and spheres (b).

The insert in Fig. 8b shows an excellent agreement with this prediction, except close to the rough wall due to deviations of solid fraction and inertial number from their value in the bulk, as was previously shown in $[8,22]$.

\section{The "dead zone" of polyhedra near the susbtrate}

\section{Specific features}

The bottom part of the flowing layer, about 5 diameters thick, in assemblies of polyhedra particles, has several seemingly anomalous properties. Solid fraction (Fig. 6) and coordination number (Fig. 7) are larger than in the bulk, velocity gradient and angular velocities decrease to zero at the bottom susbtrate (Figs. 8 and 9), and so does inertial number $I$ (Fig. 12). Normal stress differences (Fig. 11) also behave differently in that region.

As the flow is almost arrested near the substrate, we refer to this bottom layer as the dead zone. Such a nearly arrested zone was experimentally observed for flows of angular grains down a rough inclined plane[64]. If its upper boundary is conventionnally placed at the maximum of the profile of $\left|\omega_{x}\right|$ (Fig. 9a), its thickness then slightly decreases for larger $\theta$, from $5 d\left(\theta=35.7^{\circ}\right)$ down to $4 d\left(\theta=38^{\circ}\right)$. The microstructure of the dead zone (see Fig. 13) exhibits no apparent positional or orientational ordering of polyhedra, beyond the short range correlations induced by excluded volume and face to face contacts. 


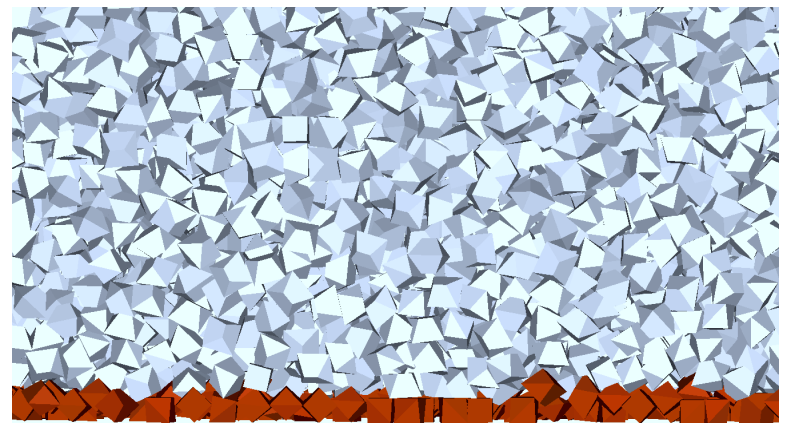

FIG. 13: (Color online) Bottom part of flowing polyhedron layer, as seen from the side. $\theta=35.7^{\circ}$.

\section{The different contact types between polyhedra}

Structural and mechanical properties of the contact network exhibit characteristic differences between the dead zone and the bulk material. Fig. 14 displays profiles of coordination numbers for $\theta=35.7^{\circ}$ and $\theta=38^{\circ}$, distinguishing between simple $\left(Z_{s}\right)$, double $\left(Z_{d}\right)$ and triple contacts $\left(Z_{t}\right)$, as defined in Sec. II C, in connection with Fig. 4. All three coordination numbers increase within

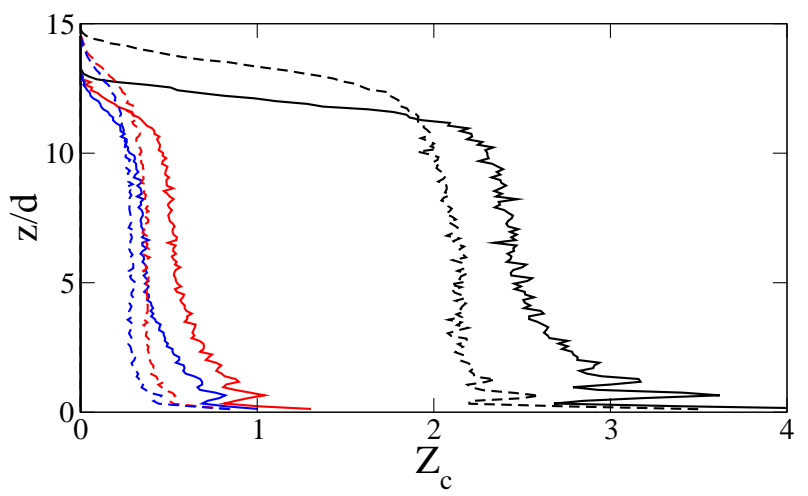

FIG. 14: (Color online) Profiles of coordination numbers $Z_{s}$ (black curves), $Z_{d}$ (red/gray curves), and $Z_{t}$ (blue/light gray curves), for $\theta=35.7^{\circ}$ (solid lines) and $\theta=38^{\circ}$ (dashed lines).

the dead zone on approaching the bottom surface, but the proportion of face to face contacts increases too, most notably for lower slope angle $\theta$. As $Z_{s}$ increases by about $20 \%, Z_{t}$ nearly doubles through the dead zone, and consequently ratio $Z_{t} /\left(Z_{s}+Z_{d}+Z_{t}\right)$ grows from 0.11 in the bulk to about 0.19 near the substrate for $\theta=35.7^{\circ}$.

To assess the mechanical importance of different contact types, one may write down shear stress $\sigma_{y z}$ as the sum of the contributions of simple $\left(\sigma_{y z}^{s}\right)$, double $\left(\sigma_{y z}^{d}\right)$ and triple $\left(\sigma_{y z}^{t}\right)$ contacts:

$$
\sigma_{y z}=\sigma_{y z}^{s}+\sigma_{y z}^{d}+\sigma_{y z}^{t} .
$$

Fig. 15 shows profiles of corresponding contributions to the stress ratio, $\mu_{\xi}^{*}=\sigma_{y z}^{\xi} / \sigma_{z z}, \xi$ denoting contact type $s$, $d$ or $t$. From Fig. 10, one may deduce that friction coef-
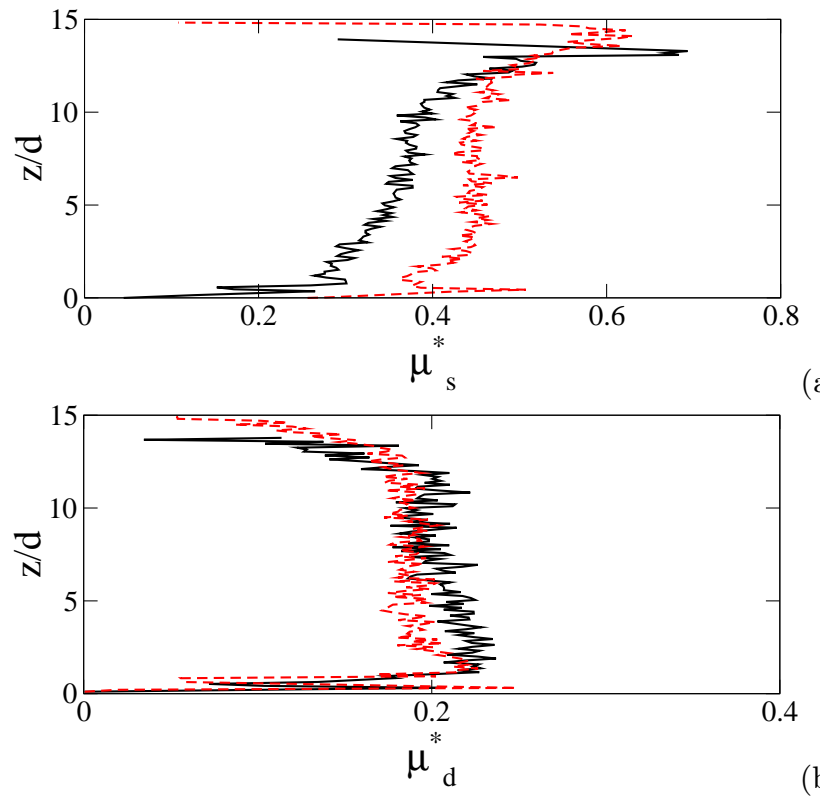

a)

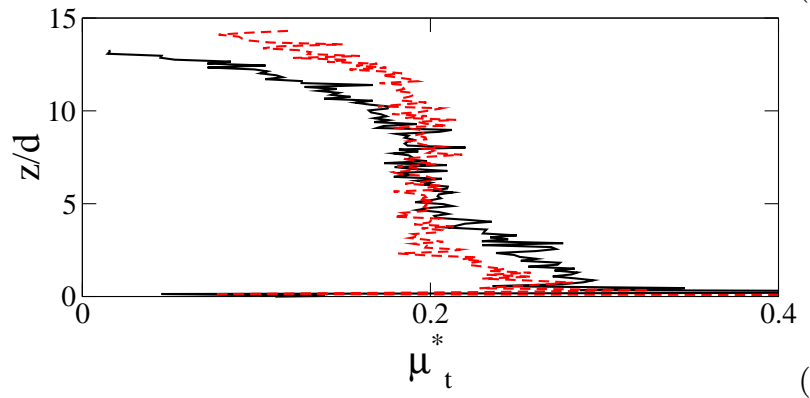

(b)

FIG. 15: (Color online) Profiles of $\mu_{s}^{*}(\mathrm{a}), \mu_{d}^{*}$ (b) and $\mu_{t}^{*}$ (c), for $\theta=35.7^{\circ}$ (solid black line) and $\theta=38^{\circ}$ (dashed red/gray line).

ficient $\mu^{*}=\mu_{s}^{*}+\mu_{d}^{*}+\mu_{t}^{*}=\sigma_{y z} / \sigma_{z z}$ is constant through the whole layer, including the dead zone. The data of Fig. 15 thus reveal a significant increase of the contribution of face to face (triple) contacts to the internal friction coefficient in the dead zone, which is correlated to a decrease of the contribution of simple contacts. Remarkably, $\mu_{t}^{*}$ and $\mu_{s}^{*}$ are nearly equal at the bottom of the dead zone for $\theta=35.7^{\circ}$, while the density of simple contacts is about three times as large as that of triple ones.

The normal stress ratio profiles displayed in Fig. 16 show that the network of face to face contacts is the major contributor to the increase of the normal stress differences in the dead zone.

In the dead zone, the prevalence of face to face contacts, which considerably restrict relative motions of contacting grains is likely responsible for the gradual vanishing of velocity gradients. 
measured effective friction coefficient values $\left(\mu^{*}=\tan \theta\right)$, mean solid fraction and inertial number (measured for $z / d$ between 8 and 15 for spheres, between 6 and 12 for polyhedra).

The calculated inertial number values range from 0.05 to 0.31 for spheres, and from 0.54 to 0.98 for polyhedra. The highest values indicate the beginning of the collisionnal regime whereas the lowest values correspond to flow stop.

The steady flows of polyhedra down a smooth frictional substrate provide two other points for much smaller value of inertial number.

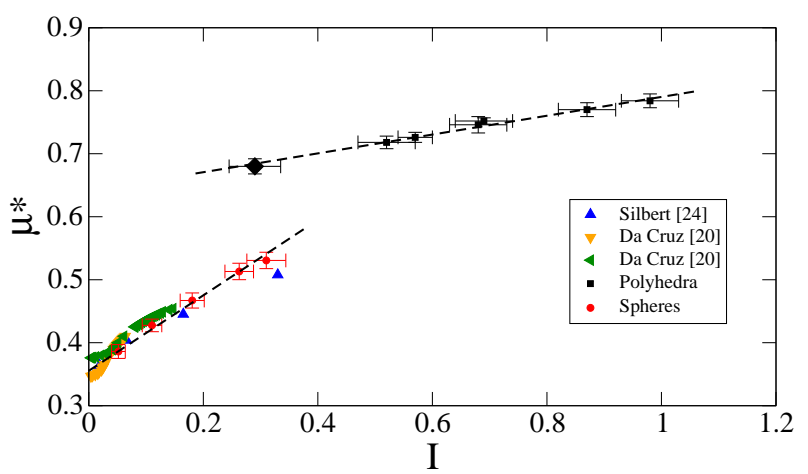

FIG. 19: (Color online) Friction law for spheres (red) and for polyhedra (black). The diamond (other symbol) corresponds to smooth frictional substrate.

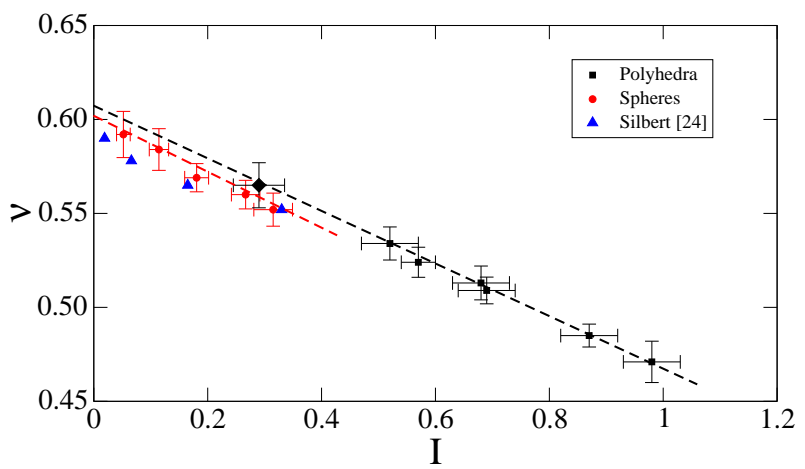

FIG. 20: (Color online) Dilation law for spheres (red) and for polyhedra (black). The diamond (other symbol) corresponds to smooth frictional substrate.

Effective friction and solid fraction dynamic laws corresponding to the present simulations are shown respectively on Fig. 19 and 20, both figures showing results for spheres and polyhedra. Error bars denote the standard deviation calculated from $\nu$ (Fig. 6), $\mu^{*}$ (Fig. 11) and $I$ (Fig. 12) measurements. These laws can be fitted by linear expressions (2) and (3), for which we measure $\nu_{\max }=0.601, \mu_{\min }^{*}=0.35, a=0.56$ and $b=0.15$ for spheres and $\nu_{\max }=0.607, \mu_{\min }^{*}=0.635, a=0.14$ and $b=0.13$ for polyhedra. It can be observed that $\mu_{\min }^{*}$ values compare very well with $\tan \theta_{m}$ values given in Tab. II (respectively 0.344 for spheres and 0.624 for polyhedra).
These values evidence a strong quantitative grain shape effect on the friction law.

Interestingly, in the case of polyhedra, the points deduced from the flow down a smooth frictional substrate (in the whole layer) nicely coincide with the linear law identified for the flow down a rough substrate (in the bulk). This is consistent with the fact that the constitutive law is intrinsic to the material and does not depend on the boundary condition. Varying the value of the friction coefficient $\mu_{W}$ at the smooth substrate might allow to measure the constitutive law in a larger range of inertial number.

These results correspond to particular values of the friction coefficient $(\mu=0.4)$ and the restitution coefficients $\left(e_{N}=e_{T}=0\right)$. Measurements with disks evidenced a significant influence of the contact friction coefficient on the effective macroscopic friction coefficient, but no influence of the restitution coefficient value $[3,17]$. Similar findings are expected for both spheres and polyhedra, in addition to a shape effect for polyhedra, and this will have to be confirmed in the future.

Our results for spheres are compared to other ones in the literature [20, 24] in Fig. 19 for the friction law (for which both experimental results [20] and numerical ones deduced from [24] are shown) and in Fig. 20 for the dilation law (with the numerical data of [24]).

Flow height $H$ and tilt angle $\theta$ dependencies of the velocity profile may be derived from the friction law as:

$v_{y}(z)=\frac{2 \sqrt{\nu(\theta) g \cos \theta}}{3 b d}\left(\tan \theta-\mu_{\min }^{*}\right)\left(H^{3 / 2}-(H-z)^{3 / 2}\right)$.

Thus, with $V=\frac{1}{H} \int_{0}^{H} v_{y}(z) d z$ denoting the mean velocity, one readily obtains:

$$
V(\theta, H)=\frac{2 \sqrt{\nu(\theta) g \cos \theta}}{5 b d} H^{3 / 2}\left(\tan \theta-\mu_{\min }^{*}\right),
$$

whence (given the values of $\theta$ )

$$
V(\theta, H) \propto H^{3 / 2}\left(\theta-\theta_{m}\right) .
$$

This expression is compatible with experimental and numerical measurements [18, 19]. Ref. [19] fits experimental data with expression $V(\theta, H) \propto \frac{H^{3 / 2}}{H_{\text {stop }}(\theta)}$, hence from (4) a similar factor of $\theta-\theta_{m}$.

\section{CONCLUSION}

The influence of grain angularity on the properties of dense flows down a rough inclined plane has been investigated by comparison between sphere and polyhedral grain assemblies. Upon clarification of the flow conditions, the dense permanent flow regime has been analyzed in details through the examination of various flow quantities, then rheology aspects have been discussed. 
We find both sphere and polyhedra assemblies to obey the flow start and flow stop laws identified in [19], with quite different parameters, as flow thresholds for polyhedra correspond to much larger tilt angles. An analysis of dense permanent flow regime for polyhedra and for spheres reveals the following similarities and differences between spherical and polyhedral grains. In the bulk, both systems exhibit uniform solid fraction, coordination numbers and inertial number. An increase of tilt angle $\theta$ entails faster flows with larger shear rates and inertial effects, smaller solid fraction and coordination number. Velocity profiles assume Bagnoldian convex shapes and angular vecocities tend to decrease at growing distance $z$ from the substrate. Solid fraction and internal friction coefficient vary linearly with inertial number $I$ for $I$ varying from $5 \times 10^{-2}$ to 0.3 (for spheres) or between 0.5 and 1 (for polyhedra). Quantitatively, polyhedral grain flows occur for higher inertial number (for the tested inclined plane configuration and $H / d$ of order 10), are faster and have lower solid fraction than spherical grain flows. For a given flow height, the range of tilt angles covered by the dense permanent flow regime is considerably narrower for polyhedra than for spheres. Some qualitative differences are also observed between spherical and polyhedral grains. Near a rough substrate, polyhedral grain samples do not structure in parallel layers and a dead zone appears, 4 or 5 diameters thick, where the flow is anomalously slow, the velocity profile is concave, and the density of faceto-face contacts, as well as their contribution to stresses and internal friction, notably increase. Limited tests of the sensitivity of the dead zone phenomenon to substrate roughness reveal that it is hardly sensitive to the size and spacing of asperities but disappears for smooth, frictional substrates (in which case, within the narrow inclination angle interval corresponding to steady flow, a velocity discontinuity is observed at the substrate, with a smooth, convex velocity profile throughout the granular layer).

Whereas normal stress components $\sigma_{x x}, \sigma_{y y}, \sigma_{z z}$ are about the same for spheres (with $\sigma_{x x}$ slightly smaller than the other two in the bulk of the material), polyhedral grain flows exhibit significant normal stress differences, which increase in the "dead zone". These features correlate with the important mechanical role of face to face contacts within this region.

More detailed investigations of the microstructure of packings of polyhedral particle, exploring, in particular, ranges of orientational correlations and additional properties of contact networks should shed more light on the observed behaviour. Such perspectives should be addressed in future work.

\section{Acknowledgments}

We gratefully acknowledge many interesting discussions with Frédéric Dubois and Farhang Radjaï at various stages of this study.
[1] O. Pouliquen and F. Chevoir, Comptes-Rendus Physique 3, 163 (2002).

[2] GDR MIDI, Euro. Phys. J. E 14, 341 (2004).

[3] F. da Cruz, S. Emam, M. Prochnow, J.-N. Roux, and F. Chevoir, Phys. Rev. E 72, 021309 (2005).

[4] G. Lois, A. LemaÓtre, and J. M. Carlson, Phys. Rev. E 72, 051303 (2005).

[5] P. Jop, Y. Forterre, and O. Pouliquen, Nature 441, 727 (2006).

[6] F. Chevoir, J.-N. Roux, F. da Cruz, P. Rognon, and G. Koval, Powder Tech. 190, 264 (2008).

[7] Y. Forterre and O. Pouliquen, Annu. Rev. Fluid Mech. 40, 1 (2008).

[8] F. Chevoir, Granular flows (in French) (Laboratoire Central des Ponts et Chaussées - Collection Etudes et Recherches des Laboratoires des Ponts et Chaussées, 2009).

[9] F. RadjaÔ and S. Roux, in The physics of granular media, edited by H. Hinrichsen and D. Wolf (Wiley-Vch, Berlin, 2004), pp. 165-187.

[10] I. Goldhirsch, Annu. Rev. Fluid Mech. 35, 267 (2003).

[11] A. Lemaître, J.-N. Roux, and F. Chevoir, Rheologica Acta 48, 925 (2009).

[12] T. Hatano, Phys. Rev. E 75, 060301 (2007).

[13] P.-E. Peyneau and J.-N. Roux, Phys. Rev. E 78, 011307 (2008).

[14] G. Koval, A. Corfdir, J.-N. Roux, and F. Chevoir, Phys. Rev. E 79, 021306 (2009).
[15] P. Rognon, J. N. Roux, M. NaaÔm, and F. Chevoir, J. Fluid Mech. 596, 21 (2008).

[16] C. Cassar, M. Nicolas, and O. Pouliquen, Phys. Fluids 17, 103301 (2005).

[17] J.-N. Roux and F. Chevoir, Dimensional Analysis and Control Parameters, chap. 8, pp. 199-232, in [76] (2011).

[18] E. Azanza, Two-dimensional granular flows down an inclined plane (in French), vol. SI5 (Collection Etudes et Recherches des Laboratoires des Ponts et Chaussées, Paris, 1998).

[19] O. Pouliquen, Phys. Fluids 11, 542 (1999).

[20] F. da Cruz, Ph.D. thesis, Ecole Nationale des Ponts et Chaussées (2004), http://pastel.paristech.org/946.

[21] F. Chevoir, M. Prochnow, J. Jenkins, and P. Mills, in Powders and grains 2001, edited by Y. Kishino (Balkema, Rotterdam, 2001), pp. 373-376.

[22] M. Prochnow, Ph.D. thesis, Ecole Nationale des Ponts et Chaussées (2002), http://pastel.paristech.org/321.

[23] F. da Cruz, M. Prochnow, J.-N. Roux, and F. Chevoir, in Powders and Grains 2005, edited by R. Garcia-Rojo, H. J. Herrmann, and S. McNamara (A.A. Balkema, Leiden, The Netherlands, 2005), pp. 361-364.

[24] L. E. Silbert, D. Ertas, G. S. Grest, T. Halsey, D. Levine, and S. J. Plimpton, Phys. Rev. E 64, 385 (2001).

[25] A. Daerr and S. Douady, Nature 399, 241 (1999).

[26] T. P?schel and V. Buchholtz, Phys. Rev. Lett. 71, 3963 (1993). 
[27] U. Mutze, Granular Matter 8, 185 (2006).

[28] X. Zhang and L. Vu-Quoc, Mech. Mat. 32, 115 (2000).

[29] L. Vu-Quoc, X. Zhang, and O. Walton, Computer Methods in Applied Mechanics Engineering 187, 483 (2000).

[30] C. Olson, C. Reichhardt, M. McCloskeyand, and R. Zieve, Europhys. Lett. 57, 904 (2002).

[31] M. Lu and G. R. McDowell, Granular Matter 9, 69 (2007).

[32] C. O'Sullivan and J. Bray, in Powders and Grains 2005, edited by R. Garcia-Rojo, H. J. Herrmann, and S. McNamara (A.A. Balkema, Leiden, The Netherlands, 2005).

[33] F. Cantelaube, Y. Limon-Duparcmeur, D. Bideau, and G. Ristow, J. Phys. I 5, 581 (1995).

[34] X. Oviedo, K. Sab, and P.-E. Gautier, in Colloque physique et mécanique des matériaux granulaires, edited by F. Chevoir and J. N. Roux (Laboratoire Central des Ponts et Chaussées, Paris, 2000).

[35] A. Schinner, Granular Matter 2, 35 (1999).

[36] H. Matuttis, Granular Matter 1, 83 (1998).

[37] H. Matuttis, S. Luding, and H. Herrmann, Powder Tech. 109, 278ñ (2000).

[38] F. Alonso-Marroquin and H. Herrmann, Phys. Rev. E 66, 021301 (2002).

[39] E. Azéma, Ph.D. thesis, Université Montpellier II (2007), http://tel.archives-ouvertes.fr/tel-00184757.

[40] D. Petit, F. Pradel, G. Ferrer, and Y. Meimon, in Powders and grains 2001, edited by Y. Kishino (Balkema, Rotterdam, 2001), pp. 425-428.

[41] E. Dimnet, Ph.D. thesis, Ecole Nationale des Ponts et Chaussées (2002).

[42] G. Saussine, Ph.D. thesis, UniversitÈ Montpellier 2 (2004).

[43] A. Donev, F. Stillinger, P. Chaikin, and S. Torquato, Phys. Rev. Lett. 92, 255506 (2004).

[44] S. Sacanna, L. Rossi, A. Wouterse, and A. P. Philipse, J. Phys. Condens. Matter 19, 376108 (2007).

[45] I. Zuriguel, T. Mullin, and J. Rotter, Phys. Rev. Lett. 98, 028001 (2007).

[46] I. Zuriguel and T. Mullin, Proc. Roy. Soc. Lond. A 464, 99 (2008).

[47] R. C. Hidalgo, I. Zuriguel, D. Maza, and I. Pagonabarraga, Phys. Rev. Lett. 103, 118001 (2009).

[48] E. Azéma and F. Radjaï, Phys. Rev. E 81, 051304 (2010).

[49] T. Kanzaki, M. Acevedo, I. Zuriguel, I. Pagonabarraga, D. Maza, and R. Hidalgo, Euro. Phys. J. E 34, 133 (2011).

[50] E. Azéma and F. Radjaï, Phys. Rev. E 85, 031303 (2012).

[51] B. Saint-Cyr, C. Voivret, J.-Y. Delenne, F. Radjaï, and P. Sornay, in Powders and Grains 2009, edited by M. Nakagawa and S. Luding (2009), vol. 1145 of AIP Conference Proceedings, pp. 389-392.

[52] B. Saint-Cyr, C. Voivret, J.-Y. Delenne, F. Radjai, and P. Sornay, Phys. Rev. E 84, 041302 (2011).

[53] E. Azéma, N. Estrada, and F. Radjaï, Submitted to Phys Rev E (2012).

[54] N. Estrada, E. Azéma, F.Radjai, and A. Taboada, Phys. Rev. E 84, 011306 (2011).

[55] C. Hogue and D. Newland, Powder Tech. 78, 51 (1994).

[56] T. P?schel and V. Buchholtz, J. Phys. I 5, 1431 (1995).

[57] V. Buchholtz, T. Poschel, and H.-J. Tillemans, Physica
A 216, 199 (1995).

[58] P. Cleary and M. L. Sawley, Applied Mathematical Modelling 26, 89 (2002).

[59] P. Langston, M. Al-Awamleh, F. Fraige, and B. Asmar, Chem. Eng. Sci. 59, 425 (2004).

[60] J. de Jaeger, in Powders and Grains, edited by J. Biarez and R. Gourves (Balkema, Rotterdam, 1989), pp. 319322.

[61] J. de Jaeger, in Powders and Grains, edited by C. Thornton (Balkema, Rotterdam, 1993), pp. 321-325.

[62] T. Börzsönyi, T. C. Halsey, and R. E. Ecke, Phys. Rev. Lett. 94, 208001 (2005).

[63] T. Börzsönyi and R. E. Ecke, Phys. Rev. E 76, 031301 (2007).

[64] I.S.Aranson, L.S.Tsimring, Malloggi, and E. Clement, Phys.Rev.E 78, 031303 (2008).

[65] C. S. Campbell, Phys. Fluids 23, 013306 (2011).

[66] R. Artoni, A.-C. Santomasoa, M. Go, and P. Canu, Phys. Rev. Letters 108, 238002 (2012).

[67] P. Cleary, Powder Tech. 179, 144 (2008).

[68] F. Y. Fraige, P. A. Langston, and G. Z. Chen, Powder Tech. 186, 224 (2008).

[69] J. Wang, H. S. Yu, P. Langston, and F. Fraige, Granular Matter 13, 1 (2011).

[70] S. Mack, P. A. Langston, C. Webb, and T. York, Powder Tech. 214, 431 (2011).

[71] D. Hohner, S. Wirtz, and V. Scherer, Powder Tech. (2012).

[72] G. Saussine, C. Cholet, P. Gautier, F. Dubois, C. Bohatier, and J.-J. Moreau, Comput. Methods Appl. Mech. Eng. 195, 2841 (2006).

[73] E. Nezami, Y. Hashash, D. Zaho, and J. Ghaboussi, Computers and Geotechnics 31, 575 (2004).

[74] E. G. Nezami, Y. M. A. Hashash, D. Zaho, and J. Ghaboussi, Int. J. Num. Anal. Meth. Geotech. 30, 783 (2006).

[75] E. Azéma, F. Radjaï, and G. Saussine, Mech. Mat. 41, 729 (2009).

[76] F. Radjaï and F. Dubois, eds., Discrete-element Modeling of Granular Materials (ISTE-Wiley, 2011).

[77] Http://www.uepg.eu/uploads/documents/pub-39_enuepg_annual_review_2010-2011_en.pdf.

[78] C. Tourenq and D. Fourmaintraux, Bulletin des Laboratoires des Ponts et Chaussées 51, 61 (1971), in French.

[79] H. Moreaud, Revue GÈnÈrale des Routes 447, ? (1969), in French.

[80] A. Panis and C. Tourenq, in Proc. 6th Int. Congress IAEG, Theme VII-3, edited by D. Price (Balkema, Rotterdam, 1990), p. 17, in French.

[81] J. Garnier, J. Robert, and R. Campanac, Bulletin des Laboratoires des Ponts et Chaussées numĖro spÈcial XIV, 77 (1984), in French.

[82] J. Garnier and J. Robert, Bulletin des Laboratoires des Ponts et Chaussées 104, 67 (1979), in French.

[83] Y. Descantes, Y. Fosse, and G. Ehret, Materials and Structures (RILEM) 39, 3 (2006).

[84] F. Hommand-Etienne, Y. Song, and H. Rapin, in Powders and Grains, edited by J. Biarez and R. Gourvès (A. A. Balkema, Rotterdam, 1989), pp. 135-141.

[85] L. Tocquer, S. Lavergne, Y. Descantes, and F. Chevoir, in Powders and Grains 2005, edited by R. Garcia-Rojo, H. J. Herrmann, and S. McNamara (A.A. Balkema, Leiden, The Netherlands, 2005), pp. 1345-1348. 
[86] Y. Descantes, C. Ropert, L. Tocquer, F. Gaulard, and F. Chevoir, Bulletin des Laboratoires des Ponts et Chaussées 267, 19 (2007).

[87] C. Tourenq and A. Denis, Les essais de granulats, vol. 114 (Rapport de recherche du Laboratoire Central des Ponts et Chaussées, Paris, 1982).

[88] C. S. Campbell, J. Fluid Mech. 465, 261 (2002).

[89] W. R. Ketterhagen, J. S. Curtis, and C. R. Wassgren, Phys. Rev. E 71, 061307 (2005).

[90] L. Silbert, G. G. S., R. Brewster, and A. J. Levine, Phys. Rev. Lett. 99, 068002 (2007).

[91] P. Jop, Y. Forterre, and O. Pouliquen, J. Fluid Mech. 541, 167 (2005)

[92] R. Laniel, Ph.D. thesis, Université Montpellier II (2007), http://perso.univrennes1.fr/romain.laniel/memoire_these.pdf.

[93] J.-J. Moreau, Eur. J. Mech. A 13, 93 (1994).

[94] J. Moreau and M. Jean, Engineering systems design and analysis pp. 201-208 (1996).

[95] F. Radjaï and V. Richefeu, Mechanics of Materials 41, 715 (2009).

[96] G. Lois, A. LemaÓtre, and J. M. Carlson, Europhys. Lett. 76, 318 (2006).

[97] E. Azéma, F. Radjaï, R. Peyroux, F. Dubois, and G. Saussine, Phys. Rev. E 74, 031302 (2006).

[98] E. Azéma, F. Radjaï, F. Peyroux, R, and G. Saussine, Phys. Rev. E 76, 011301 (2007).

[99] N. Estrada, A. Taboada, and F. Radjaï, Phys. Rev. E 78, 021301 (2008).
[100] E. Azéma, F. Radjaï, R. Peyroux, V. Richefeu, and G. Saussine, Euro. Phys. J. E 26, 327 (2008).

[101] V. Visseq, A. Martin, D. Iceta, E. Azéma, D. Dureisseix, and P. Alart, Comput Mech DOI 10.1007/s00466012-0699-5 (2012)

[102] F. Radjai, Physics of dry granular media (Kluwer Academic Publishers (Dordrecht/Boston/London), 1997), chap. Multicontacts dynamics, p. 305.

[103] V. Richefeu, S. El Youssoufi, E. Azéma, and F. Radjai, Powder Tech. 190, 258263 (2009).

[104] F. Dubois and M. Jean, in Actes du sixi Ëme colloque national en calcul des structures - CSMA-AFM-LMS (2003), vol. 1, pp. 111-118.

[105] F. Dubois and M. Jean, The non smooth contact dynamic method: recent LMGC90 software developments and application (Springer Berlin / Heidelberg, 2006), vol. 27 of Lecture Notes in Applied and Computational Mechanics, pp. 375-378, URL http://dx.doi.org/10. 1007/3-540-31761-9_44.

[106] M. L?tzel, S. Luding, and H. J. Hermann, Granular Matter 2, 123 (2000)

[107] I. Agnolin and J.-N. Roux, Phys. Rev. E 76, 061302 (2007).

[108] J. J. Moreau, in Friction, Arching, Contact Dynamics, edited by D. E. Wolf and P. Grassberger (World Scientific, Singapore, 1997), pp. 233-247.

[109] R. A. Bagnold, Proc. Roy. Acad. London A 225, 49 (1954) 